\title{
Multiobjective Optimization Approach to Localization of Ectopic Beats by Single Dipole: Case Study
}

\author{
Jana Svehlikova ${ }^{1}$, Jan Zelinka ${ }^{1}$, Milan Tysler ${ }^{1}$, Peter Tino ${ }^{2}$ \\ ${ }^{1}$ Institute of Measurement Science, SAS, Bratislava, Slovakia \\ ${ }^{2}$ School of Computer Science, University of Birmingham, Edgbaston, UK
}

\begin{abstract}
A novel reliability parameter was suggested for the inverse solution using a single dipole for localization of the origin of an undesired ventricular activity from clinical data.

Body surface potential maps (BSPMs) were measured for several minutes on the patient with premature ventricular contractions and implanted cardiostimulator. The measured signal was segmented into ECG beats that were further clustered according to their morphology. Assuming a normal distribution of the signals within the cluster a parameter ñreliability scoreò $(R S)$ evaluating the distribution of the inversely computed dipole moments was suggested. Then the optimal inverse solution (dipole) was estimated in the multi-objective optimization setting through simultaneous optimization of both the relative residual error (RRE) between the input BSPM and the map computed by the inverse dipole and the RS parameter.

The method was applied on BSPMs measured for 5 minutes of imposed pacing (498 beats). The inclusion of the reliability score lead to improvement of the localization error from $16 \mathrm{~mm}$ (RRE only) to $11 \mathrm{~mm}(R R E+R S)$ and from 13 to $9 \mathrm{~mm}$ for a homogeneous and inhomogeneous torso, respectively.
\end{abstract}

\section{Introduction}

A simplified method of electrocardiographic imaging (ECGI) is the inverse solution using a single dipole that was introduced for finding an origin of the undesired heart electrical activity which is localized in a small area of the myocardium [1]. In general, ECGI aims to reconstruct the heart electrical activity on the whole surface of ventricles during the heartbeat. The attempts of several research groups were summarized recently in [2]. Because the inverse problem is ill-posed its solution requires some form of regularization, selection of which is one of the specific tasks of the problem [3]. Clinical applicability of
ECGI reconstruction of activation as well as the repolarization phase of the heartbeat cycle was studied and demonstrated also in [4].

In our previous works [1], localization of the dipole best representing the input BSP map was derived according to the criterion of the minimal relative residual error between the input BSP map and the BSP map computed from the dipole estimated by the inverse solution. In this study, we introduce an additional criterion to complement the residual error, so that the localization of the undesired ventricular contraction is performed in the multiobjective optimization setting.

\section{Materials and methods}

Body surface potentials mapping lasting several minutes was performed on the male patient (age 57) with cardiostimulator and suffering by premature ventricular contractions (PVC). ECG signals were recorded in three modes of the cardiostimulator function: (1) cardiostimulator worked in the usual setting of patientôs everyday life, (2) no cardiostimulator intervention and (3) an electrode implanted in the right ventricle near apex was paced with the frequency of 100 beats per minute. The measurements were carried out in cooperation with The National Institute for Cardiovascular Diseases in Bratislava on the ProCardio8 system developed in our laboratory [5] using 128 electrodes in 16 strips placed regularly on the patientôs torso.

After the measurement was completed the patient underwent a CT scan of the torso together with the used electrodes positioned on the torso. The CT images were later processed by the specialized software Tomocon [9] to create an individual 3D model of torso surface including the surfaces of the heart, the heart cavities, and lung lobes. Positions of the measuring electrodes were also captured as well as the position of the tip of the implanted electrode from cardiostimulator (Figure 1). The patient signed the informed consent to the whole procedure.

During the long-term measurements, the repeated ECG 
signal can be contaminated by respiration or superposition of preceding heart cycle as it was pointed out also in [6]. Thus the baseline of the measured signal was determined by setting a zero-point in such time interval where the isoelectric line was present. Then the baseline was computed using the cubic spline fitting the defined zero points in all measured signal.

The measured signal was segmented into ECG beats as the defined time interval around each R-peak of the ECG signal. Then the ECG beats were further clustered according to their morphology, and an averaged beat was computed for each cluster by ensemble averaging of ECG segments aligned by R-peak time instant.

First, the inverse solution using a single dipole as the equivalent generator was computed by the previously described methodology [1]. Possible positions of the inverse result were predefined inside the model of myocardial volume in $2 \mathrm{~mm}$ grid. Then, for the averaged signals in BSP map in each time instant within the early 20 $\mathrm{ms}$ of activation the dipole best representing the input BSP map was computed according to the criterion of the minimal relative residual error (minRRE) between the input BSP map and the BSP map computed from the dipole estimated by the inverse solution. The position of the dipole in the time instant when the minRRE was minimal was considered as the most probable position of the PVC origin.

Second, the statistical properties of signals in the cluster representing the stimulated ECG were used as follows. Considering a dipole as an equivalent cardiac generator in the predefined position, the BSP map (p) on the torso can be computed in each time instant by the linear equation:

$$
\mathbf{p}=\mathbf{T d} \text {, }
$$

where $\mathbf{T}$ is the transfer matrix computed by a boundary element method, $\mathbf{d}$ is the vector of the corresponding dipole moments.

The averaged signal in the BSP map was obtained from several individual beats. We assumed that at each time instant the signal values were normally distributed. Assuming the input signal $\mathbf{p}$ in the linear equation (1) is normally distributed with mean $\mu$ and covariance $\mathbb{E}$, the dipole moments in vector $\mathbf{d}$ are also normally distributed with mean

$$
\mathbf{m}=\mathbf{T}^{+} \boldsymbol{\mu}
$$

and covariance

$$
\mathbf{C}=\mathbf{T}^{+} \mathbf{E}\left(\mathbf{T}^{+}\right) \hat{\mathbf{Q}}
$$

where $\mathbf{T}^{+}$is pseudoinverse od the transfer matrix $\mathbf{T}$ and ôis the matrix transposition operator. The covariance matrix $\mathbf{C}$ characterizes spread of the distribution of the inversely computed dipole moments for the selected position in the heart, given the measured data on the torso. From $\mathbf{C}$ we calculate ñeliability scoreò (RS) for the corresponding localization position in the heart as the sum of the eigenvalues of $\mathbf{C}$ (sum of variances along the decorrelated axes). The score quantifies the induced uncertainty of dipole moments, given the variability around the averaged
BSP signal in the cluster.

The RS parameter was evaluated in each time instant within the first $20 \mathrm{~ms}$ of paced signal for 1000 best localizations obtained solely based on the RRE criterion. To find the optimal solution with respect to both RRE and RS parameters, the Pareto front [7] in the RRE-RS space was computed for each time instant. Then the ñtotalò Pareto front over all such Pareto fronts for the whole time interval was created. The balance between the optimized RRE and RS was determined at the knee point of the total Pareto front and the corresponding localization was selected as the ñoptimalò overall localization. The localization error between this localization and the known position of the top of the pacing electrode was calculated as the Euclidean distance between the two points.

The inverse solution was computed assuming the homogeneous as well as inhomogeneous torso model.

\section{Results}

The inverse solution to a single dipole was evaluated either using our original single criterion of minRRE within the studied time interval or by adding the RS criterion and optimizing both criteria.

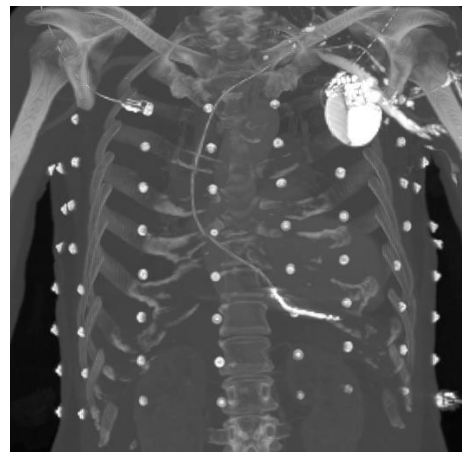

Figure 1. CT scan of the patientôs torso, where the actual positions of the recording electrodes as well as the position of the stimulating catheter are available.

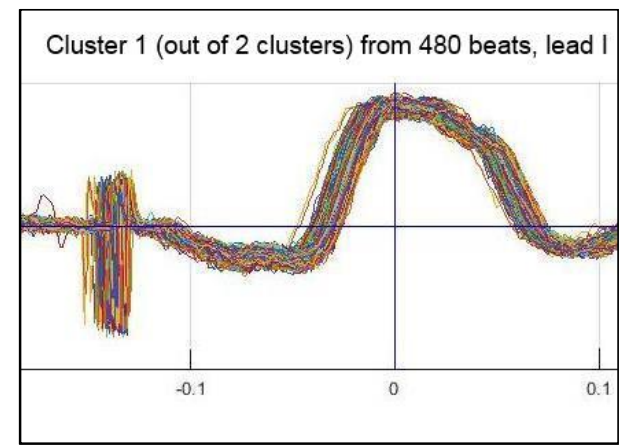

Figure 2. The cluster of ECG signals in the standard lead I evoked by pacing catheter, aligned by R-peak. 
Five minutes recording was performed during the imposed pacing with the frequency of 100 beats per minute. After the clustering procedure, a cluster with 480 beats representing the paced signal was obtained (Figure 2 ). Because of a possible influence of the metal cardiostimulator body on the measured signals, the signals from 12 electrodes placed nearest to the device were not used for the inverse computation, thus we employed BSP map with 116 measured points.

The stimulating impulse was clearly visible in the ECG signal, so the $20 \mathrm{~ms}$ time interval just after the impulse was examined.

For each time instant, the inverse solution to single dipole was computed in more than 135.000 predefined positions in the myocardial volume. Then for each time instant, the RS parameter was computed for 1000 positions with the smallest RRE value (see figure 3 ). The resulting Pareto front in the RRE-RS space is shown as red dots in Figure 3. The total Pareto fronts over the whole studied time interval for homogeneous and inhomogeneous torso model are presented in Figures 4 and 5, respectively. The arrows point to the results chosen by minRRE criterion only and by optimization of the two criteria (knee).

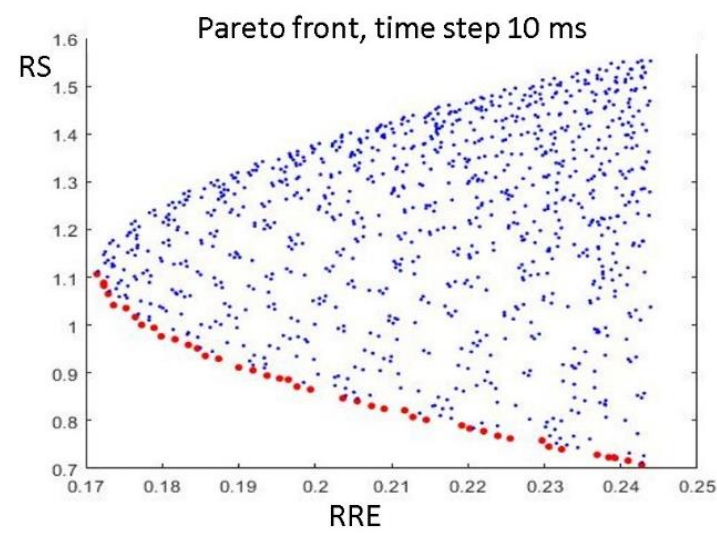

Figure 3. Pareto front (red dots) from the 1000 results with minimal RRE value (blue dots) in a time instant.

Localization driven solely by the minimal RRE resulted in LE of $16 \mathrm{~mm}$ and $13 \mathrm{~mm}$ for homogeneous and inhomogeneous torso models, respectively. When joint minimization of RRE and RS was considered (knee point on the total Pareto front), the LE improved to $11 \mathrm{~mm}$ and 9 $\mathrm{mm}$, respectively.

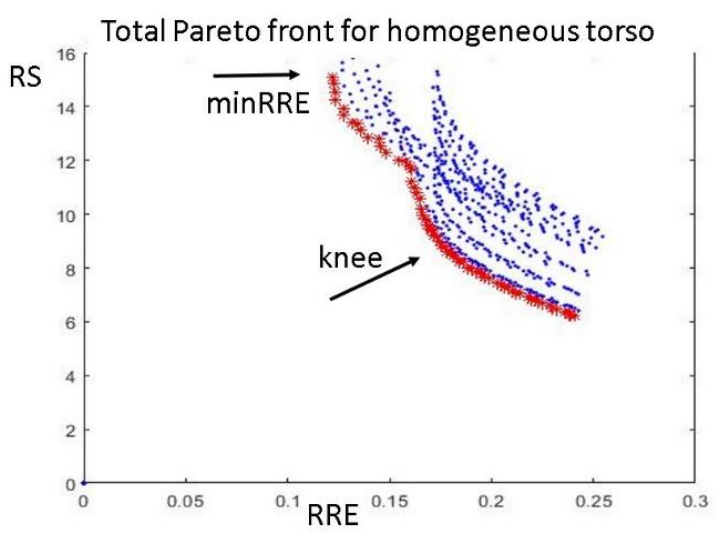

Figure 4. Total Pareto front (red) from all Pareto fronts (blue) within the studied $20 \mathrm{~ms}$ time interval for homogeneous torso model.

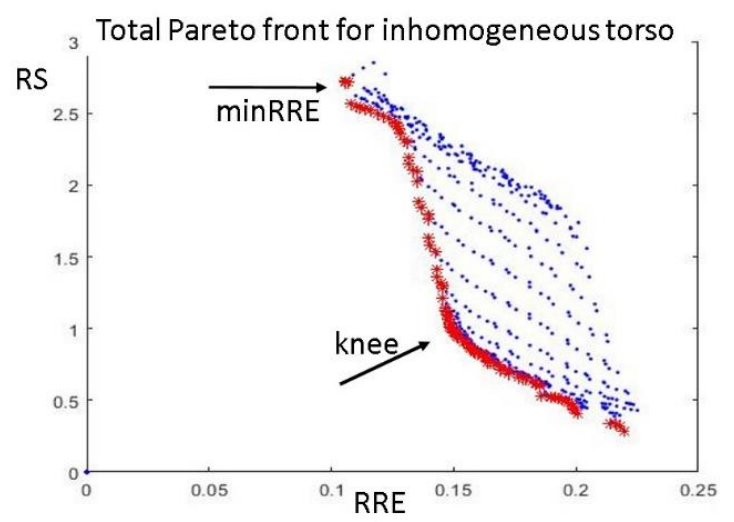

Figure 5. Total Pareto front (red) from all Pareto fronts (blue) within the studied $20 \mathrm{~ms}$ time interval for inhomogeneous torso model.

\section{Discussion and conclusion}

We presented a modified inverse solution using a single dipole to find the origin of an undesired ventricular contraction...

A single dipole used as an equivalent electrical generator for the inverse solution can be considered as a geometrical constraint of the inverse problem, which is in general ill-posed [1]. The proposed RS parameter serves as an additional localization constraint. It utilizes basic statistical properties of the measured experimental data and transforms them to statistical properties of the induced dipole moments. Therefore, the measurements lasting several minutes are desirable for the method, so that the statistics could be reliably estimated. The statistical properties of the experimental data were used also for the inverse reconstruction of the epicardial potentials over/during the whole heartbeat cycle in more sophisticated probabilistic approaches such as in [8]. 
The study is limited to one BSP measurement with a well-defined position of stimulating catheter near the right ventricular apex. More such experiments, as well as a variety of the stimulating point position, is needed to evaluate the possible positive impact of the presented method on the inverse solution.

\section{Acknowledgements}

This work was supported by the research grant 2/0125/19 from the VEGA Grant Agency in Slovakia and by the grant APVV-14-0875 from the Slovak Research and Development Agency.

\section{References}

[1] Svehlikova J, Teplan M, Tysler M. Geometrical Constraint of Sources in Noninvasive Localization of Premature Ventricular Contractions. J Electrocardiol 2018;51:370ї 7. doi:10.1016/j.jelectrocard.2018.02.013.

[2] Cluitmans M, Brooks DH, MacLeod R, Dössel O, Guillem MS, van Dam PM, et al. Validation and Opportunities of Electrocardiographic Imaging: From Technical Achievements to Clinical Applications. Front Physiol 2018;9:1ї 19. doi:10.3389/fphys.2018.01305.
[3] Johnston PR. Accuracy of Electrocardiographic Imaging Using the Method of Fundamental Solutions. Comput Biol Med 2018. doi:10.1016/j.compbiomed.2018.09.016.

[4] Bear LR, Bouhamama O, Cluitmans M, Duchateau J, Walton RD, Abell E, et al. Advantages and Pitfalls of Noninvasive Electrocardiographic Imaging. J Electrocardiol 2019. doi:10.1016/j.jelectrocard.2019.08.007.

[5] Kadanec, J. Zelinka, J. Bukor, G. Tysler M. ProCardio 8 System for High Resolution ECG Mapping. Meas. 2017, Proc. 11th Int. Conf. Smolenice, Slovakia, 2017, p. 263 ï 6.

[6] Coll-Font J, Brooks DH. Tracking the Position of the Heart From Body Surface Potential Maps and Electrograms. Front Physiol 2018;9:1727. doi:10.3389/fphys.2018.01727.

[7] Kalyanmoy D. Dominance and Pareto-Optimality. MultiObjective Optim. using Evol. Algorithms. 1 st ed,

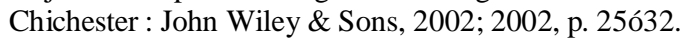

[8] Onak ON, Serinagaoglu Dogrusoz Y, Weber GW. Effects of a Priori Parameter Selection in Minimum Relative Entropy Method on Inverse Electrocardiography Problem. Inverse Probl Sci Eng 2018;26:877ї 97. doi:10.1080/17415977.2017.1369979.

[9] https://tatramed.sk/en/tomocon-workstation/

Address for correspondence.

Jana Svehlikova

Institute of Measurement Science, Slovak Academy of Sciences Dubravska cesta 9, 84104 Bratislava, Slovakia jana.svehlikova@savba.sk 